\title{
Does Intra-articular Tranexamic Acid Decrease Blood Loss in Total Hip Arthroplasty?
}

\author{
Ganesan G Ram ${ }^{1}$, Phagal Varthi Vijayaraghavan ${ }^{2}$
}

\begin{abstract}
Background: Blood is the nurturing liquid that delivers oxygen to the cells of the body and removes carbon dioxide and waste from the body. Blood is considered as liquid gold. Total hip replacement causes blood loss and in turn needs blood transfusion. The aim of the study is to find the role of intra-articular tranexamic acid (TXA) in control of blood loss in total hip replacement surgeries.

Materials and methods: A prospective study of 50 patients who underwent total hip replacement surgery. The study group and control group patients were selected through the card method. In the study group after the skin closure, $2 \mathrm{~g}$ of TXA was injected through the drain tube and drain tube was clamped for 1 hour and then released. Blood loss during the first and second postoperative periods from suction drain was calculated. Results: Four hundred and fifty-five milliliters was the mean first postoperative day blood collected in the drain tube in the control group while study group had $283 \mathrm{~mL}$. Similarly, the second day mean postoperative day drain was $89 \mathrm{~mL}$ in control group and $25 \mathrm{~mL}$ in study group. The mean first and second postoperative day blood loss was statistically significant between the groups.

Discussion: There was a considerable reduction of transfusions in postoperative period; hence, there was a reduced hospitalization cost and less risk of transmitted infections and transfusion reactions. There is a negligible risk of thrombosis in patients who had intravenous TXA not the intra-articular TXA.

Conclusion: Intra-articular TXA is an effective tool in controlling blood loss and reducing blood transfusions in hip replacement surgeries.

Keywords: Blood loss, Blood transfusion, Hip replacement, Thrombosis, Tranexamic acid.

Journal of Orthopedics and Joint Surgery (2020): 10.5005/.jp-journals-10079-1022
\end{abstract}

\section{INTRODUCTION}

Blood is the nurturing liquid that delivers oxygen to the cells of the body and removes carbon dioxide and waste from the body. It is a sort of connective tissue that comprises red blood cells, platelets, and white blood cells suspended in a fluid plasma network. Seven to eight percent of body weight is made up of blood. ${ }^{2}$ Blood is considered as liquid gold. Total hip replacement causes blood loss and in turn needs blood transfusion. Blood and blood products should be used in minimum and only needed during surgeries. There is an ongoing research to decrease this intraoperative and postoperative blood loss. Blood-sparing strategies are one element of the rapid recovery protocols used for joint replacement surgery. Tranexamic acid has its rightful place in this context. There are many studies regarding the use of TXA in total knee replacement but very few about total hip replacement. ${ }^{3,4}$

\section{Aim and Objective}

The aim and objective of the study is to find the role of intra-articular TXA in control of blood loss in total hip replacement surgeries.

\section{Materials and Methods}

A prospective study of 50 patients who underwent total hip replacement surgery from June 2018 to February 2020. Institutional ethics committee approval was obtained before the commencement of the study. Informed consent and written consent were obtained from all the participants of the study. The inclusion criteria were patients who underwent total hip arthroplasty for traumatic and non-traumatic indications having hemoglobin $>10 \mathrm{~g} / \mathrm{dL}$ and having normal coagulation profile. The exclusion criteria were patients with bleeding disorders, thrombotic
${ }^{1}$ Department of Orthopaedics, Velammal Medical College, Madurai, Tamil Nadu, India

${ }^{2}$ Department of Orthopaedics, Sri Ramachandra University, Chennai, Tamil Nadu, India

Corresponding Author: Ganesan G Ram, Department of Orthopaedics, Velammal Medical College, Madurai, Tamil Nadu, India, Phone: +91 9444779755, e-mail: ganesangram@yahoo.com

How to cite this article: Ram GG, Vijayaraghavan PV. Does Intra-articular Tranexamic Acid Decrease Blood Loss in Total Hip Arthroplasty? J Orth Joint Surg 2020;2(2):49-51.

Source of support: Nil

Conflict of interest: None

episodes, taking antiplatelet drugs, and hematological disorders. There were two groups of patients, study and control groups. The patients who received intra-articular TXA were the study group. The blood collected in the drain was considered as the primary outcome measurement while postoperative blood transfusion was considered as the secondary endpoint. The study group and control group patients were selected through the card method. Single surgeon did all the surgeries and posterior approach was used for all the cases. Vario drain was placed before the capsular closure and then wound closed in layers. The patient is given generation intravenous cephalosporin for the first 5 days. The patient is nursed in absolute aseptic conditions in the postoperative ward with the limb protected by an abduction pillow placed between the legs and a small pad beneath the knee to maintain it in slight flexion. Drains are removed at the end of 48 hours. Drain tips are sent for microbiological examination. The preoperative and postoperative 
protocols were same for all the patients except for the intra-articular TXA use in the study group.

In the study group after the skin closure, $2 \mathrm{~g}$ of TXA ( $1 \mathrm{~g}$ of TXA is equivalent to 1 ampoule of $10 \mathrm{~mL}$ ) was injected through the drain tube and drain tube was clamped for 1 hour and then released. We had 26 male and 24 female patients. Patients were in equal numbers in both the groups ( 25 patients). Blood loss during the surgery was calculated by two methods and the mean was taken as intraoperative blood loss. One was by measuring intraoperative hematocrit values while the other was by manual observation from drain, surgical mops, and from the operation field. Postoperative blood loss calculated from collection from suction drain during the first and second postoperative days. ${ }^{5}$ Any blood transfusion intraoperatively or in the postoperative period was noted. The postoperative hematocrit was also checked. The actual blood loss (ABL) was calculated from a modification of the gross formula given below:

$$
\mathrm{ABL}=B V[\operatorname{Hct}(i)-\operatorname{Hct}(f)] / \operatorname{Hct}(\mathrm{m})]
$$

where $\mathrm{BV}$ is the blood volume and equals body weight $(\mathrm{kg}) \times 70$ $\mathrm{mL} / \mathrm{kg}$; Hct (i), Hct $(\mathrm{f}$, ) and Hct $(\mathrm{m})$ are the initial, final, and mean (of the initial and final) hematocrit, respectively. No transfusions were done according to $A B L$. For each patient, the numerical difference in blood loss (DIFF-BL) equals ABL-EBL. The average blood loss (AVE-BL) is also calculated, which is the mean of EBL and ABL. Blood transfusion was considered when hemoglobin is $<8 \mathrm{~g} / \mathrm{L}$. We used no routine screening for thrombosis, but one patient who had clinically suspected thromboembolic complications in the first 6 weeks was investigated using contrast venography.

\section{Results}

The average intraoperative blood loss in the control group and study group were 860 and $856 \mathrm{~mL}$, respectively. Four hundred and fifty-five milliliters were the first postoperative day blood collected in the drain tube in the control group while study group had $283 \mathrm{~mL}$. Similarly, the second postoperative day drain was $89 \mathrm{~mL}$ in control group and $25 \mathrm{~mL}$ in study group. There was nearly $41 \%$ less postoperative drain on the first postoperative day in study group, which is statistically significant. The study group patients needed 10 transfusions while patients in control group had 30 blood transfusions.

\section{Discussion}

Blood was a limited asset with a constrained timeframe of realistic usability and was related with high handling costs. Use of blood and blood products needs basic audit to recognize areas of overuse and in this manner decrease patient to patient and hospital costs. Dreariness and mortality may result from either an immunologically intervened response or a transmitted contamination. Tranexamic acid, epsilon-aminocaproic acid (EACA), and aprotinin were three antifibrinolytics agents that can be used to decrease perioperative bleeding in total hip replacement and other surgical procedures. ${ }^{6-8}$ Tranexamic acid is a synthetic derivative of the amino acid lysine and binds the 5 -lysine binding sites on plasminogen and inhibits plasmin formation and displaces plasminogen from the fibrin surface. ${ }^{9}$ In our study, we had used TXA only to evaluate it use in control of blood loss in total hip replacement surgeries.

In our study, TXA, given intra-articular at the end of the operation, was an efficient and cost-effective way to reduce blood loss in total hip replacement surgeries. Intravenous TXA was
Table 1: $t$-test for the first postoperative day blood loss in total hip replacement

\begin{tabular}{lllll}
\hline Group & $n$ & Mean & Std. dev & $\begin{array}{l}\text { Std. error } \\
\text { mean }\end{array}$ \\
\hline Control & 25 & 455.2 & 207.3 & 44.3 \\
Study & 25 & 283.5 & 77.05 & 13.4 \\
\hline
\end{tabular}

Table 2: Mann-Whitney test for blood loss in total hip replacement

\begin{tabular}{lc}
\hline & Postoperative day 1 \\
\hline Mann-Whitney U & 122.000 \\
Wilcoxon W & 447.000 \\
$Z$ & -3.581 \\
Asy. Sig (two-tailed) & 0.000 \\
\hline
\end{tabular}

not preferred for the study as there were complications, such as pulmonary embolism, uterine obstruction, venous and arterial obstruction, increased risk of leukemia, and adenocarcinoma of hepatic duct associated with intravenous use of TXA. ${ }^{10,11}$ Blood transfusion in the study group was significantly less when compared to the control group. In our study of total hip arthroplasty, the average first postoperative blood loss in control group was $455 \mathrm{~mL}$ and study group was $283 \mathrm{~mL}$. Similar results were obtained by Benoni but the difference between our study and his study was that our study patients had TXA injection intra-articularly while Benoni patients had it intravenously. ${ }^{12}$ From Tables 1 and 2, it was clear that the blood loss was statistically significant when TXA was used. Blood loss during the surgery was calculated by two methods and the mean was taken as intraoperative blood loss. One was by measuring intraoperative hematocrit values while the other was by manual observation from drain, surgical mops, and from the operation field. We found that the blood loss calculations by hematocrit values were found to be more accurate than the observation technique. The study group had a significant reduction in the postoperative drain collection. Our study group, patients who had intra-articular TXA injection had nearly $41 \%$ reduction of postoperative drain in the first 24 hours.

There was a considerable reduction of transfusions in postoperative period; hence, there was a reduced hospitalization cost and less risk of transmitted infections and transfusion reactions. We did not routinely conduct screening for thrombosis in our study. Even though there were literature support for the correlation of TXA and thrombosis, none of the patient in our study group developed deep vein thrombosis. Studies have shown that thrombosis prophylaxis using low-molecular-weight heparin delayed the onset of thromboses to the fourth or fifth postoperative day. ${ }^{13}$ For these reasons, we consider it highly unlikely that we would had been able to show any thrombogenic effect of the administration of TXA. Even though some literature supporting thrombosis as the side effect of TXA when used intravenous or intramuscular, we provocate the use intra-articularly making this complication unlikely. There is a negligible risk of thrombosis in patients who had intravenous TXA not the intra-articular TXA. ${ }^{12}$ The limitations of the study were small sample size and routine contrast venogram was not done for all patients, which in turn can have a negative impact in diagnosing asymptomatic deep vein thrombosis.

\section{Conclusion}

Intra-articular TXA is an effective tool in controlling blood loss and reducing blood transfusions in hip replacement surgeries. 


\section{References}

1. McSwiney MM, O'Farrell D, Joshi GP, et al. Blood transfusion in total hip athroplasty: guidelines toeliminate overtransfusion. Can J Anaesth 1993;40(3):222-226. DOI: 10.1007/BF03037034.

2. Liumbruno G, Bennardello F, Lattanzio A, et al. Recommendations for the transfusion of red blood cells. Blood Transfus 2009;7(1):49. DOI: 10.2450/2008.0020-08

3. Ollivier JE, Van Driessche S, Billuart F, et al. Tranexamic acid and total hip arthroplasty: optimizing the administration method. Ann Transl Med 2016;4(24):530. DOI: 10.21037/atm.2016.11.53.

4. Zhu J, Zhu Y, Lei $P$, et al. Efficacy and safety of tranexamic acid in total hip replacement: a PRISMA-compliant meta-analysis of 25 randomized controlled trials. Medicine 2017;96(52). DOI: 10.1097/ MD.0000000000009552.

5. Ram GG, Suresh P, Vijayaraghavan PV. Surgeons often underestimate the amount of blood loss in replacement surgeries. Chin J Traumatol 2014;17(4):225-228.

6. Yamasaki S, Masuhara K, Fuji T. Tranexamic acid reduces postoperative blood loss in cementless total hip arthroplasty. J Bone Joint Surg Am 2005;87(4):766-770. DOI: 10.2106/JBJS.D.02046.

7. Hardy J, Belisle S, Dupont C, et al. Prophylactic tranexamic acid and epsilon-aminocaproic acid for primary myocardial revascularization.
ATS 1998;65(2):371-376. DOI: 10.1016/s0003-4975(97) 01016-3.

8. Harley B, Beaupré L, Jones A, et al. The effect of epsilon aminocaproic acid on blood loss in patients who undergo primary total hip replacement: a pilot study. Can J Surg 2002;45(3):185-190.

9. Pabinger I, Fries D, Schöchl H, et al. Tranexamic acid for treatment and prophylaxis of bleeding and hyperfibrinolysis. Wien Klin Wochenschr 2017;129(9-10):303-316. DOI: 10.1007/s00508-017-1194-y.

10. Mayman DJ. Intravenous versus topical tranexamic acid in total knee arthroplasty: both effective in a randomized clinical trial of 640 patients. J Bone Joint Surg Am 2018;100(12):1023-1029. DOI: 10.2106/ JBJS.17.00908.

11. North WT, Mehran N, Davis JJ, et al. Topical vs intravenous tranexamic acid in primary total hip arthroplasty: a double-blind, randomized controlled trial. J Arthroplasty 2016;31(5):1022-1026. DOI: 10.1016/j. arth.2015.11.003.

12. Benoni G. Reduction of blood loss in orthopaedic surgery. In orthopaedic proceedings. Br Editor Soc Bone Joint Surg 2006;88(No. Supp_I):17-17.

13. Myers SP, Kutcher ME, Rosengart MR, et al. Tranexamic acid administration is associated with an increased risk of posttraumatic venous thromboembolism. J Trauma Acute Care Surg 2019;86(1): 20-27. DOI: 10.1097/TA.0000000000002061. 